\title{
Development and Research of School-Based Curriculum of Primary Mathematics Focusing on Mathematics Activities
}

\author{
Huanhuan Xiong ${ }^{1, a}$, Youhong $\mathrm{Xu}^{1}$, Weixing $\mathrm{CaO}^{2, \mathrm{~b}}$ and Hongying Huang ${ }^{1, \mathrm{c}}$ \\ ${ }^{1}$ School of Mathematics, Zhejiang Ocean University, Zhejiang, China \\ ${ }^{2}$ School of Civil Engineering, Zhejiang Ocean University, Zhejiang, China \\ axionghuanhuan1995@163.com, b373789910@qq.com, '30540726@qq.com
}

Keywords: School-based curriculum; Mathematics activities; Primary Mathematics

\begin{abstract}
With the enactment of three-level curriculum management policy in our country, the development of school-based curriculum has become the task that schools and teachers must face. How to develop school-based curriculum of primary school mathematics has put forward higher requirements for schools and teachers, and there are many problems we need to explore and study both in theory and in practice. First, this paper describes the importance of development for school-based curriculum of primary mathematics. In addition, it reveals existing major drawbacks in today's school-based mathematics curriculum for primary students, finally attempting to improve students' enthusiasm in learning mathematics based on taking full advantage of school-based mathematics curriculum for primary students, also provide a good reference for its future development.
\end{abstract}

\section{Introduction}

As we all know, the characteristics of pupils' thinking is concrete operations, in other words, the operation of mathematical logic needs the help of specific object or manifestations. The mathematics learning of pupils should base on a large number of specific operational activities then gradually transits to abstract symbolic computation.[1-3] The current mathematics textbooks of elementary schools have already paid attention to the close contact between mathematics and life and established the content of research studies of comprehensive practice activities, but in practical teaching, many figurative still bear certain limitations. For example, the daily life for families with children is filled with the mathematical figure everywhere, from finding the home location, the route back to home to the placing of furniture, use of plastic bags, and calculation of major household anniversaries and etc. Although kids learn these things in mathematics lessons, however, it is required to train kids to obtain the ability to discover mathematics during their daily life.

In view of the above situation, I made my own school-based curriculum - "math activities" From the meaningful life experiences of children, students could turn their life experience into abstract mathematical model then explain and apply it[4-7]. Thereby it enables students to gain mathematical understanding, meanwhile, it helps improve and develop their mathematical thinking, problem-solving ability, attitudes and values and so on. School-based curriculum of mathematics not only needs to develop the course stamped with "mathematical activity", but also adapt to national mathematics curriculum.[8-10].

\section{Significance of Development of School-Based Curriculum of Primary Mathematics}

With further development of curriculum reform, the teaching method of teachers changes, thus realizing transfer from teaching textbook to teaching with textbook. As a result, based on respect for textbook, we should explore and expand textbook according to practical demand, and giving ply to the function of textbook to the largest extent, finally realizing optimal teaching combination, thus developing students' thoughts.

In general, when it comes to teaching, people will know one-sidedly that maths is abstract symbol and solve problems. Exam-oriented education makes maths teachers design excessive exercises 
training targeting at exams in maths study. In daily maths, they tend to reinforce central subject status of maths study while neglecting the educational function of it as to each student, thus leading to indifference of many students as to study. Many students lack excellent emotional experience and attention on personality in maths study, thus it is difficult for them to form happy experience, and those vivid ideas and delectable maths aesthetic factors disappear. It is indicated in investigation that the values and functions of maths course endowed through mathematical ideas, historical materials, culture, and aesthetics level as to human quality and personality cultivation.

The new mathematics education should promote the overall development and quality of students through the Mathematics Teaching. As the new values, quality-oriented education emphasizes the cultural factors of math education, and attaches importance to cultural values of math education as basic content requirements of quality-oriented education. The course is set up to discuss the development of mathematics teaching, the role of mathematics as well as the value of mathematics in broad cultural background of mathematics, and the content of the course makes up for some deficiencies in mathematics teaching materials and can stimulate students 'interest in learning and develop the students' mind.

School-based curriculums are characterized by its strong focus. The precondition of the curriculum development is in pursuit of the interest and the need of students. The course is set up according to the students' practical situation, so it can and should take into account the interests of our students. Therefore, it helps students develop their expertise.

\section{Problems in Development of School-based Curriculum of Primary Mathematics}

\subsection{Teachers' ability for course design needs to be improved}

Teachers are better at designing the program of activities, and accustomed to describe a specific part with teaching language in the design process. In the long teaching career, they get used to this way to implement the course, copy materials, and turn the language of instruction into teaching language. Once teachers participate in this session of course design, they often do not know how to set the course objectives, and they even suspect the meaning of the discussion of curriculum goals. In the preparation of teaching materials, they often use the existing teaching materials of mathematics of other countries, As a result, it is similar to nationally standard mathematics courses to certain degree. Development of school-based curriculum is a subject for teachers in every aspect, and teachers' ability for curriculum design needs to be improved.

\subsection{Eager to present materials}

Teachers are most familiar with teaching materials, rather than courses. In the teachers' opinion, writing textbooks and making researchers are considered to be achievements. They are eager to present teaching materials so that they put a lot of energy and time into writing teaching materials, while ignoring the studies of the whole system of curriculum frameworks and teaching materials. In fact, teaching materials is just one aspect of the final presentation, instead of the main aspects. For course, the most important part is the syllabus, but we always overlook it. Lacking guidance of syllabus, there will be no specific direction and provisions for writing of teaching materials.

\subsection{Guidance of expert needs a process for transformation}

During the school curriculum development, experts' guidance is essential. However, the ideas of experts' and teachers' are very different. Experts get used to find the essence of the phenomenon from the chaos, find the law from the concrete practice, thus to find out where the value is for promotion. However teachers focus on specific teaching content that is the school-based curriculum itself and the specific form of teaching. This contradiction between the experts and teachers results in difficulties in the guidance process. Therefore, the experts should give full consideration to the actual situation of teachers in elementary schools, allow a process of transformation in their own guidance, so as to unify experts' and teachers' powers, reach a consensus and change practices. 


\section{Features of Activity Maths Course in Elementary School}

The teaching contents and methods in maths activity course in elementary school is confirmed by aspects like individualized teaching, and students' interests and habits. However, in general, the teaching of activity course should manifest educational, scientific, practical, ideological, interesting, automatic, opening, and hierarchical feature while integrated with consciousness of national condition, scientific technology, competition, economy, democracy, and cooperation, and cultivate students' ability in thinking, operation, creation, flexibility, communication and organization, finally realizing its teaching objectives.

\subsection{Educational feature}

Educational feature refers to distinct educational orientation of activity course content, referring to maths activity makes education of ideology and morality, as well as excellent behavioral habits and so on. At present, the current situation and development of reform and opening to the outside world and national economy integrated with consciousness of national conditions, thus motivating learning activity of students, defining learning objective and activating desire for knowledge learning. For example, penetrate rational shopping among students in Supermarket shopping activity combined with children's day activity, and make ideological education of saving among students.

\subsection{Practicalness}

Practicalness refers to connecting teaching contents with students' practice in life such as teaching tool making, teaching practice and problem solving and so on, allowing students to operate by them, cultivate operation ability. There is a common feature for the several courses, referring to experiment contents arising in nature course. Iron stand, and balance as well as other experiment instruments make students operate with keen pleasure, which not only train cooperation between brain and hands, but also train students' cooperation.

\subsection{Scientificalness}

Science is according to the student's knowledge foundation and needs, choose to expand the horizons of knowledge, broaden the latest information in the field of cognitive, the contents of the latest scientific and technological achievements, infiltration of "science and technology awareness, stimulate their learning science, love science, scientific interest, so as to let the students acquire knowledge and develop intelligence. Penetrate the students' science and technology consciousness

\subsection{Openness and diversity}

Openness is referring to is carried out in class and out of class and school combining the teaching form, such as visiting, social investigation, let the students to get in touch with the society, depth of social life to feel to live in mathematics is everywhere, always to use mathematics, gradually train students to use mathematical knowledge to solve practical problems. Diversity is the subject that students choose will enable students to use their own knowledge from different angles, different levels to be resolved, and explore a variety of forms of activities.

\subsection{Interest}

Interest refers to attracting students to participate in as far as contents and methods of activity course is concerned, thus achieving entertainment in study, interest in intelligence of students in activity process. Students acquire actively and vividly knowledge. For example, the setting of activities like interesting solution to intelligent questions, maths games and intelligence contest motivate students to participate in competition with courage, thus cultivating their qualities including loyalty, firmness and confidence. Some courses design games for the whole class, but maths problems we often study are penetrated unconsciously in games, students learn actively and easily, achieving really harmonious integration in classroom atmosphere, even in the end of class, all children run for reporting actively.

\subsection{Autonomy}

Autonomy refers to delivering autonomy of study to students, allowing students to find problems by themselves, finding solution and channels to problems, cultivating students' ability in independent study and activity organization, allowing each student to perform their own personality. Through cultivation in maths activity course, children can cultivate maths consciousness in life. Just like when 
apple comes across Newton, there is exploration about gravity. The purpose of maths activity class is basically realized when our children can pay attention to maths even science in life and attempt to ask questions about details in life.

\section{Take Full Advantage of Elementary Mathematics Activities}

\subsection{Stimulate students' interest in learning mathematics}

Elementary mathematics activities should break the barriers between mathematics and life. We should start to observe and look for mathematics around us so as to guide students to discover the close connection between mathematics and life and stimulate students' interest in understanding and exploring mathematics. Life is full of mathematics, so we should use the teaching situations from life to design teaching activities, guide students to actively explore the wonders of mathematics, and stimulate their interest in mathematics. It's very important to stimulate students' interests in mathematics, as Piaget pointed out that all intelligence work depends on the interest and the interest of students has a role in promoting their intelligence. Interest is the prerequisite in mathematics education, since as long as the students have interests in mathematics they will want to explore its mystery. Therefore, interest in mathematics education is particularly important. Mathematics education in elementary school should start with life situation students are familiar with and staffs students are interested in so that it enables students learn mathematics in life and game, feel the fun of mathematics, and develop their interest in learning mathematics. For example, simulating life situation in classroom teaching could make lessons be closer to real life, strengthen students' perception, stress the key point, resolve tough problems, stimulate students' interest and diverge their thinking.

\subsection{Improve the students' ability to apply mathematics}

A lot of problems in Mathematics textbooks are mathematical problems. In order to enable students to better understand the mathematical way of thinking and improve their ability to apply mathematics, the mathematics classes are based on the theoretical knowledge in teaching system, and the mathematics practice classes put up a bridge between the theoretical knowledge and real life. First, we should bridge for students, or learning is only the endless suffering for students, so each semester should create activity classes for students; Second, we should build some bridges for the students so that they could take less detour, and we think that only one lesson in a semester is inadequate; Finally, we should provide students with some bridges of different sizes, thus, different mathematics lessons are accessible to the students. For the important knowledge useful for a long term, we can carefully prepare one or two classes like building a bridge. For example, we can carry out relevant shopping activities after learning calculating laws; we could study subjects like "watch and time", "travel and timetable" after learning time; or we can let students analyze the social issues in buying lottery after learning statistics and probability. In short, regardless of the length of the mathematical practice, It merely aims to produce a feeling that mathematics exists everywhere in life, thereby improving students' mathematics application ability.

\subsection{Improve students' learning initiative}

According to Constructivist Learning Theory, mathematical learning is a process of active construction, instead of passive acceptance, in other words, that is to construct knowledge through the interaction between the internal knowledge structure and the surrounding environment. That means that our teaching activity must be based on students 'prior knowledge and experience, then create conditions to make new learning materials to interact with students' existing cognitive structure, so that students can take the initiative to construct new mathematical cognitive structure.

Practical activities promote "learning by doing" that is to let students explore a variety of operating experience and activities, participate in the knowledge generation process and the development process, so as to discover the knowledge by themselves, experience mathematical knowledge, and develop their abilities of actively acquiring knowledge. For example: the practice of "feel game fair" 
enables students feel what is fair game and how to do it to make it fair in their personal practice. What teachers teach in the class is incomparable with the conclusion the students make from their practice.

\subsection{Effectively develop students' mathematical thinking}

Focusing on the penetration of mathematical thinking and the development of students' mathematical accomplishment is a core task of practice activities. Mathematical way of thinking refers to the method of comparative analysis, modeling approach, estimation methods, reasoning methods, transformation methods, and statistical methods and so on. For mathematics teaching in primary schools, these mathematical ideas and methods were penetrated through solving problems, so that students are unconsciously influenced with these mathematical ideas and methods. Therefore, teachers always create some situations to make the class full of discussion, inquiry and thinking atmosphere. In practice activities, teachers should get rid of the shackles of traditional teaching models and let students attempt bravely, allow students to fail, and encourage them to overcome difficulties and continue to explore. Mathematical practice activities could provide a vast space for students to explore the formation of knowledge and master the way of thinking. It helps to find the essence of internal relations by observing, manipulating, analyzing, comparing and generalizing, so as to obtain knowledge and get developed on that basis.

\section{Conclusion}

School-based curriculum development should first increase courses for teachers in the elementary school mathematics knowledge and mathematics activity training, because the student learning is diversified, learn some kinds of uniqueness of emotion, the uniqueness of personal experience, makes the student to the construction of object meaning is multidimensional, so the classroom interaction and mutual activities between teachers and students is particularly important. Secondly, we shall establish necessary management mechanism, to promote school education target and integration between teachers' potential development, and based on this platform, the school teachers form professional atmosphere of democracy, development, equality and professional culture. To avoid the phenomenon of "single-handedly hard work of teachers, curriculum reform was over." Then it can make the curriculum reform get to the student, also makes the school really get development in the curriculum reform.

\section{Acknowledgment}

2016 University Students Technology Innovation Project Proposal of Zhejiang Ocean University (Xinmiao Talents Program), and Zhejiang province higher education teaching reform research projects: The reform and practice of exploratory teaching in higher mathematics classroom (Kg2013197).

\section{References}

[1]. Ji Zhengbin. Reflections on the construction of school based curriculum and the construction of school characteristics [J]. Chinese Education Journal,2014,S4:11-12.(In Chinese)

[2]. Cheng Chenkun, Feng Haiying. On the problems and Countermeasures of school based training in Senior High School under the background of new curriculum reform [J]. Journal of Tangshan Teachers College,2012,05:145-149.(In Chinese)

[3]. He Yonghua. On the problems and Countermeasures of school based training in Senior High School under the background of new curriculum reform [J]. The way to success, 2010,10:18.(In Chinese)

[4]. Lv Lijie, Yuan Qiuhong. The logic of curriculum organization in the development of school based Curriculum [J]. educational studies,2014,09:96-103.(In Chinese) 
[5]. Me Jiali. Integration of information technology and school based curriculum development [J]. educational studies,2012,09:53-57.(In Chinese)

[6]. Wu Chunyan. Research on the present situation of school based curriculum development in the school of culture and wisdom [J]. Chinese Journal of Special Education,2013,02:31-35.(In Chinese)

[7]. Ren Shuangxi.On the importance of the development of school based curriculum in Higher Vocational Colleges [J]. Modern reading,2013,21:31.(In Chinese)

[8]. Zhou Tong, Zhang Li, Ma Lirong. On the current situation of College Students' family education and its influence on the development of family education management [J]. Education and Teaching Forum,2014,39:159-160.(In Chinese)

[9]. Zhou Chao, Liu Xianming. Research on the current situation of college students in Zhenjiang [J]. Popular literature and art, 2009,09:156-157.(In Chinese)

[10]. Feng Chunyu, Xu Jianfei. Analysis of College Students' Tutor Market [J]. Modern Enterprise Education,2010,04:144-145.(In Chinese) 